\title{
Reproductive health of young adults with physical disabilities in the U.S.
}

\author{
Annie-Laurie McRee, MPHa ${ }^{a}$ Abigail A. Haydon, MPHa,b, and Carolyn Tucker Halpern, \\ PhDa,b \\ aUNC Gillings School of Global Public Health, Department of Maternal and Child Health, 401 \\ Rosenau Hall, CB 7445, Chapel Hill, NC 27599 \\ ${ }^{b}$ Carolina Population Center, University of North Carolina at Chapel Hill, University Square, CB \\ 8120, Chapel Hill, NC 27516
}

\begin{abstract}
Objective-Previous research shows reduced cervical and breast cancer screening among women with physical disabilities. However, other indicators of reproductive health have been largely ignored. We aimed to compare the reproductive health of young adults in the U.S. with and without physical disabilities in a nationally-representative sample.

Method-Data are from 13,819 respondents aged 18-26 who participated in Waves I (19941995) and III (2001-2002) of the National Longitudinal Study of Adolescent Health (Add Health). Using logistic regression, we examined associations between physical disability and multiple reproductive health indicators including sexually transmitted infection (STI) testing, STI diagnosis, receipt of a gynecologic exam, and cervical cancer screening. Analyses were stratified by sex and adjusted for the complex study design.
\end{abstract}

Results-We identified 5.8\% of respondents as having a physical disability. In multivariate analyses, females with physical disabilities had lower odds of having a pap smear in the past 12 months than females without disabilities (OR=0.77; 95\%CI: 0.61, 0.97). Physical disability was not associated with other reproductive health indicators among females or males.

Conclusion-We found few differences in examined reproductive health indicators of young adults with and without physical disabilities, but findings suggest differences in some screening services that merit additional study.

\section{Keywords}

sexually transmitted diseases; physical disability; Papanicolau smear; reproductive health

\footnotetext{
(C) 2010 Elsevier Inc. All rights reserved.

Correspondence and requests for reprints should be sent to: Annie-Laurie McRee, MPH, UNC Gillings School of Global Public Health, Department of Maternal and Child Health, 401 Rosenau Hall, CB 7445, Chapel Hill, NC 27599, Phone: (919)265-7051, Fax: (919)966-0458, almcree@email.unc.edu.

Publisher's Disclaimer: This is a PDF file of an unedited manuscript that has been accepted for publication. As a service to our customers we are providing this early version of the manuscript. The manuscript will undergo copyediting, typesetting, and review of the resulting proof before it is published in its final citable form. Please note that during the production process errors may be discovered which could affect the content, and all legal disclaimers that apply to the journal pertain.

Conflict of interest statement

We have no conflicts of interest to disclose.
} 


\section{Introduction}

Individuals with physical disabilities are as sexually active and have the same reproductive health needs as those without a disability (Blum, et al., 2001, Cheng and Udry, 2002, Suris, et al., 1996). However, individuals with disabilities experience multiple physical and attitudinal barriers to reproductive health services (Becker, et al., 1997). Previous research shows reduced cervical and breast cancer screening among women with disabilities (Armour, et al., 2009, Nosek and Howland, 1997). Yet, other indicators of reproductive health have been largely ignored. Testing and diagnosis of sexually transmitted infections (STI) among young adults with disabilities warrant particular attention. Young people aged 15-24 have the highest rates of many STIs (Weinstock, et al., 2004). Individuals with a physical disability are less likely to receive sexuality education (Cheng and Udry, 2002), which may place them at greater risk of STIs. The purpose of this study was to compare multiple reproductive health indicators among young adults with and without physical disabilities in a nationally-representative sample. We hypothesized that young adults with physical disabilities would be less likely to receive reproductive health care and would have poorer reproductive health outcomes during young adulthood compared to their peers without physical disabilities.

\section{Methods \\ Data}

We used data from the National Longitudinal Survey of Adolescent Health (Add Health), a nationally representative survey of youth in the United States in grades 7-12 during the 1994-95 school year. Add Health utilized a complex, school-based sampling design that is described in detail elsewhere (Harris, et al., 2008). Our analytic sample consisted of respondents who participated in Wave I (1994-1995) and Wave III (2001-2002) in-home interviews, had valid sample weights, and no missing data for measures of disability or sociodemographic characteristics $(\mathrm{n}=13,819)$. The Institutional Review Board at the University of North Carolina at Chapel Hill approved all Add Health study procedures; the present analysis was deemed exempt from review.

\section{Measures}

Physical disability-We identified individuals with a physical disability using a combination of adolescent and parent report at Wave I (Cheng and Udry, 2002) and respondent report at Wave III of functional limitations and activity restriction. Component variables at Wave I assessed difficulties using limbs due to a permanent physical condition, equipment use, personal care assistance, deafness, and blindness. Wave III component variables assessed limitations in dressing, bathing, performing moderate activities, or climbing a flight of stairs, as well as difficulty hearing, deafness, and blindness. In the present analysis, we use a dichotomous measure of disability that classifies individuals as having a disability if they met criteria at either wave.

Reproductive health indicators-We assessed multiple reproductive health indicators at Wave III. For both females and males, we created a measure of STI testing based on respondent report of having been tested for one or more of 14 STIs in the past 12 months. We also examined two dichotomous measures of experiencing an STI: (1) self-report of having been diagnosed with one of 14 STIs in the past 12 months, and (2) current infection with an STI based on results of a urine specimen assay for three infections (Chlamydia trichomatis,Neisseria gonorrhoeae, and Trichomoniasis vaginalis) at the time of the interview. For females, we also created 3 variables indicating having a gynecological exam, 
cervical cancer screening (i.e., Pap smear), and abnormal Pap test result in the past 12 months.

\section{Statistical Analyses}

We examined associations between physical disability and reproductive health indicators separately for females and males using logistic regression. Multivariate models controlled for sociodemographic characteristics, insurance coverage, and years since first sex. We conducted analyses between November 2009 and February 2010, using Stata version 9.2 (Stata Corp, College Station, TX) to weight data and adjust for Add Health's complex study design.

\section{Results}

Overall, $5.8 \%$ of young adults were identified as being physically disabled (Table 1 ). The sample was approximately half female and was largely non-Hispanic white $(65.7 \%)$ or nonHispanic black (15.7\%). The mean age was 22 years $(\mathrm{SD}=1.8)$. In general, females and males had similar patterns of disability and sociodemographic characteristics but differed on some reproductive health outcomes.

Most women with physical disabilities reported having a gynecological exam $(69.7 \%)$ or a pap smear (61.8\%) within the past 12 months (Table 2). Approximately one-third said they had been tested for at least one STI during this time and a substantial number either selfreported being diagnosed with an STI (13.9\%) or tested positive on the Add Health assay $(9.3 \%)$. While women with a disability were similar to their non-disabled peers on most of the reproductive health outcomes examined, they had lower odds of having a Pap smear in the past 12 months (OR=0.77; 95\% CI: $0.61,0.97)$ in multivariate analyses.

Fewer than 1 in 5 men with a physical disability reported being tested for an STI in the past 12 months. Six percent reported an STI diagnosis and 8\% tested positive for an STI using the Add Health assay. Though they had increased odds of reporting an STI diagnosis in the past 12 months in bivariate analyses, this association was no longer statistically significant in the multivariate model.

\section{Discussion}

In this nationally-representative sample, young adults with and without physical disabilities were similar on a number of reproductive health indicators. However, in adjusted models, women with physical disabilities were significantly less likely to report receiving a Pap smear in the past year compared to those without physical disabilities. This finding supports differences in cervical cancer screening found in previous studies of women 18 and older (Armour, et al., 2009, Iezzoni, 2000, Nosek and Howland, 1997). The reason for this association warrants further investigation, especially since our analyses controlled for sexual activity and women with disabilities were equally likely to get a gynecological exam. Thus, lower rates of cervical cancer screening do not appear to be the result of difficulties accessing reproductive health care services, but may instead suggest that provider attitudes are one factor influencing the care given to women with disabilities.

In contrast to a previous study reporting increased rates of HIV testing among individuals aged 18-50 with a disability (Neri, et al., 2007), we found no associations between physical disability and STI testing in young adulthood. Differences in estimates of this relationship may be due, at least in part, to differences in outcome measures and sample populations. The previous study assessed ever having been tested for HIV while ours used a 12-month, time- 
limited measure of having been tested for several different STIs. Testing for STIs, including HIV, among individuals with a disability merits additional study.

Our study has important strengths, including a large, nationally representative sample of young adults in the U.S., and the use of biomarker data for one measure of STIs. Study limitations include reliance on self report for most outcomes and use of a general measure of physical disability that does not distinguish among type (e.g., sensory disability or motor impairment), severity, or duration. It is possible that the timing of disability acquisition could affect reproductive health outcomes and use of health care. Differential access and practice across geographic locations (Armour, et al., 2009) may also obscure associations that are context-dependent.

\section{Conclusions}

Although there are suggestive patterns, we found few statistically significant differences between young adults with and without disabilities with respect to STI testing and diagnosis. Nevertheless, differences in cervical cancer screening suggest that continued efforts on the part of health care providers and others are needed to ensure that women with disabilities receive the full range of reproductive health services, including cervical cancer screening.

\section{Acknowledgments}

We thank Gary Maslow for his valuable feedback as we developed and refined the measure of physical disability. This research uses data from Add Health, a program project designed by J. Richard Udry, Peter S. Bearman, and Kathleen Mullan Harris, and funded by a grant P01-HD31921 from the Eunice Kennedy Shriver National Institute of Child Health and Human Development, with cooperative funding from 23 other federal agencies and foundations. Special acknowledgment is due Ronald R. Rindfuss and Barbara Entwisle for assistance in the original design. Persons interested in obtaining data files from Add Health should contact Add Health, Carolina Population Center, 123 W. Franklin Street, Chapel Hill, NC 27516-2524 (addhealth@ unc.edu). No direct support was received from grant P01-HD31921 for this analysis.

\section{Role of funding source}

Dr. Halpern and Ms. Haydon's time on this project was supported by a grant from the Eunice Kennedy Shriver National Institute of Child Health and Development (1R01HD057046-01A2). The funder had no involvement in this analysis.

\section{References}

Armour BS, Thierry JAM, Wolf LA. State-Level Differences in Breast and Cervical Cancer Screening by Disability Status: United States, 2008. Womens. Health. Issues 2009;19:406-414. [PubMed: 19879454]

Becker H, Stuifbergen A, Tinkle M. Reproductive health care experiences of women with physical disabilities: A qualitative study. Arch. Phys. Med. Rehabil 1997;78:S26-S33. [PubMed: 9422004]

Blum RW, Kelly A, Ireland M. Health-risk behaviors and protective factors among adolescents with mobility impairments and learning and emotional disabilities. J. Adolesc. Health 2001;28:481-490. [PubMed: 11377992]

Cheng MM, Udry JR. Sexual behaviors of physically disabled adolescents in the United States. J. Adolesc. Health 2002;31:48-58. [PubMed: 12090965]

Harris KM, Halpern CT, Entzel P, Tabor J, Bearman PS, Udry JR. The National Longitudinal Study of Adolescent Health: Research Design. 2008

Iezzoni LI. Mobility impairments and use of screening and preventive services. Am. J. Pub. Health 2000;90:955-961. [PubMed: 10846515]

Neri SV, Bradley EH, Groce NE. Frequency of HIV Testing Among Persons with Disabilities: Results from the National Health Interview Survey, 2002. AIDS Educ. Prev 2007;19:545-554. [PubMed: 18190278] 
Nosek MA, Howland CA. Breast and cervical cancer screening among women with physical disabilities. Arch. Phys. Med. Rehabil 1997;78:39-44. [PubMed: 9014955]

Suris JC, Resnick MD, Cassuto N, Blum RW. Sexual behavior of adolescents with chronic disease and disability. J. Adolesc. Health 1996;19:124-131. [PubMed: 8863084]

Weinstock H, Berman S, Cates W Jr. Sexually transmitted diseases among American youth: incidence and prevalence estimates, 2000. Perspect. Sex. Reprod. Health 2004;36:6-10. [PubMed: 14982671] 


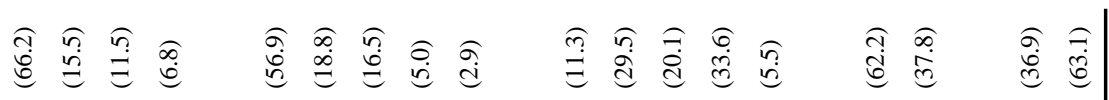

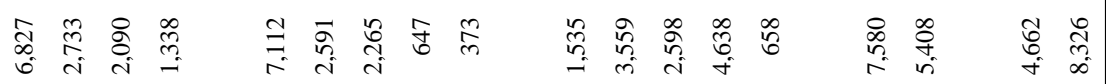

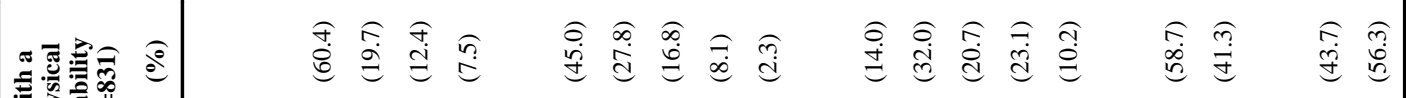

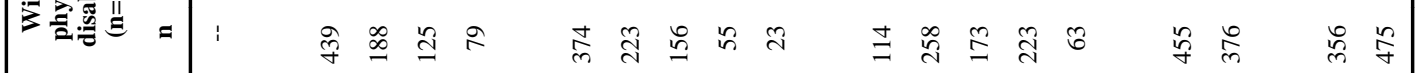
혼 


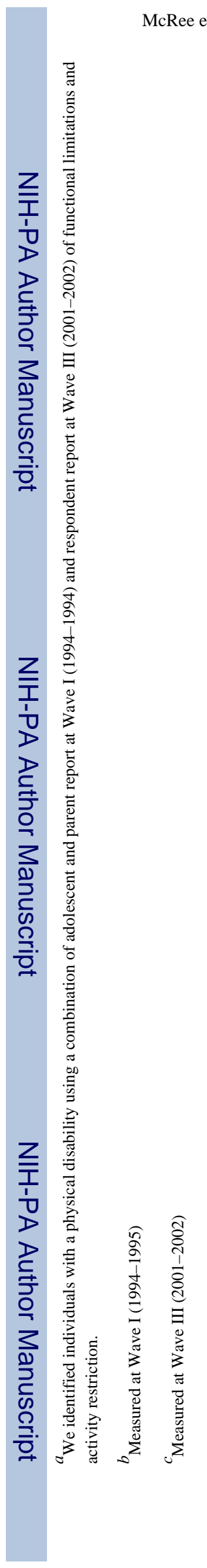

Page 7

Prev Med. Author manuscript; available in PMC 2011 December 1. 


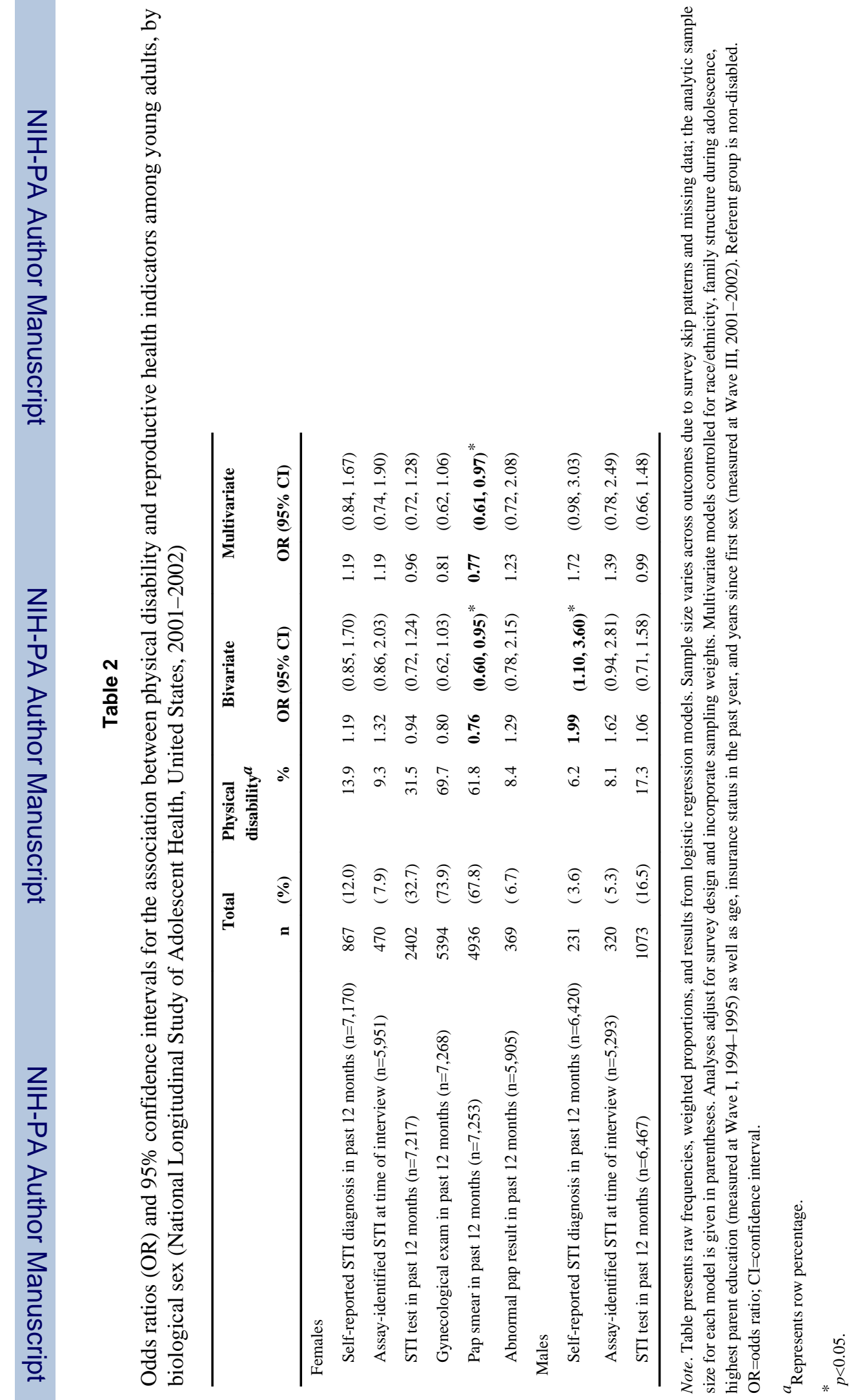

Prev Med. Author manuscript; available in PMC 2011 December 1. 